\title{
Confocal microscopy in cornea guttata and Fuchs' endothelial dystrophy
}

\author{
Auguste G-Y Chiou, Stephen C Kaufman, Roger W Beuerman, Toshihiko Ohta,
} Hisham Soliman, Herbert E Kaufman

\begin{abstract}
Aims-To report the appearances of cornea guttata and Fuchs' endothelial dystrophy from white light confocal microscopy. Methods-Seven eyes of four consecutive patients with cornea guttata were prospectively examined. Of the seven eyes, three also had corneal oedema (Fuchs' dystrophy). In vivo white light tandem scanning confocal microscopy was performed in all eyes. Results were compared with non-contact specular microscopy.

Results-Specular microscopy was precluded by corneal oedema in one eye. In the remaining six eyes, it demonstrated typical changes including pleomorphism, polymegathism, and the presence of guttae appearing as dark bodies, some with a central bright reflex. In all seven eyes, confocal microscopy revealed the presence of round hyporeflective images with an occasional central highlight at the level of the endothelium. Changes in cell morphology and size were readily appreciated. Conclusion-By comparison with specular microscopy, the hyporeflective images with an occasional central highlight seen on confocal microscopy are consistent with the presence of guttae. Confocal microscopy may confirm the diagnosis of cornea guttata and Fuchs' endothelial dystrophy by demonstrating the presence of guttae. This technique is especially valuable in cases of corneal oedema, where specular microscopy may fail to visualise the endothelium. However, specular microscopy should remain the method of choice to evaluate the endothelium, principally because it is easier to use.
\end{abstract}

(Br F Ophthalmol 1999;83:185-189)

Louisiana State

University Eye Center,

New Orleans, LA, USA

A G-Y Chiou

S C Kaufman

$\mathrm{R}$ W Beuerman

T Ohta

H Soliman

H E Kaufman

Correspondence to: Auguste G-Y Chiou, MD,

LSU Eye Center, 2020

Gravier Street, Suite B, New Orleans, LA 70112-2234, USA.

Accepted for publication 26 August 1998 microscopy allows superior image contrast and vertical and lateral resolution, compared with
Corneal guttae consist of focal accumulation of collagen at the posterior surface of Descemet's membrane. ${ }^{1}$ They are probably secreted by result of aging. Guttae are characteristic for 'uchs' endothelial dystrophy, which is a familial dominantly inherited disorder, also characerised by corneal oedema. ${ }^{2}$ Usually the condipresentation is not, although asymmetric oedema is absent and only guttae are present, the condition is called cornea guttata.

The endothelium is usually best examined conventional imaging methods. ${ }^{3-13}$ Because of its ability to focus the light source and the image on the same focal plane, it allows real time in vivo assessment of the different layers of the cornea, including the endothelial layer. Therefore, it may be an alternative method in evaluating cornea guttata or Fuchs' endothelial dystrophy.

In the current study, we analysed the appearances of cornea guttata and Fuchs' dystrophy from confocal microscopy and compare the technique with non-contact specular microscopy.
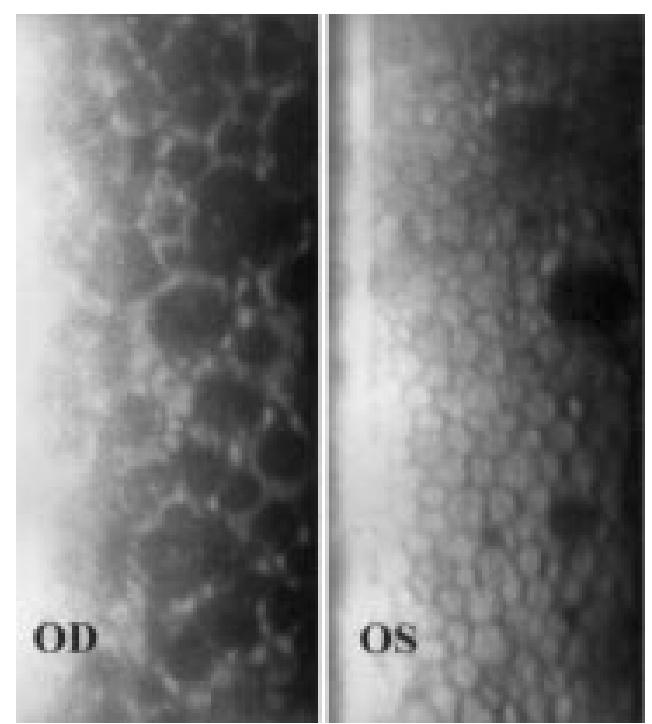

Figure 1 Patient 1. Specular microscopy in a case of cornea guttata with asymmetric presentation. Dark round bodies are more predominant in the right eye (OD). OS indicates left eye.

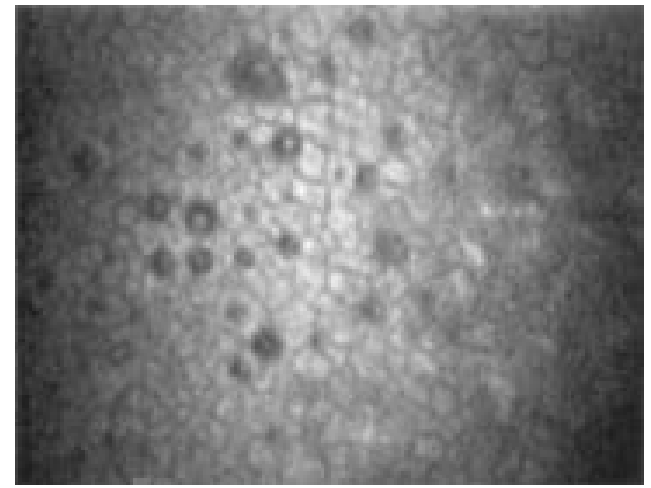

Figure 2 Patient 1. Confocal microscopy (magnification $\times 210)$ of the left eye. A few hyporeflective images with an occasional central highlight were seen at the level of the endothelium. 


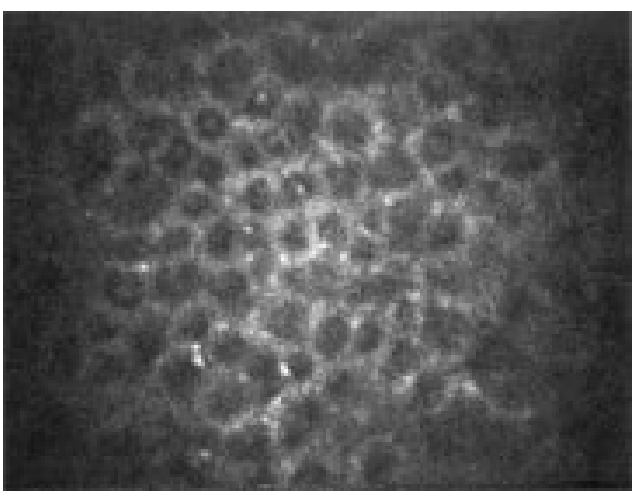

Figure 3 Patient 1. Confocal microscopy (magnification $\times 210)$ of the right eye. Compared with the left eye, significantly more hyporeflective images were seen. Hyperreflective endothelial cells were found between the lesions.
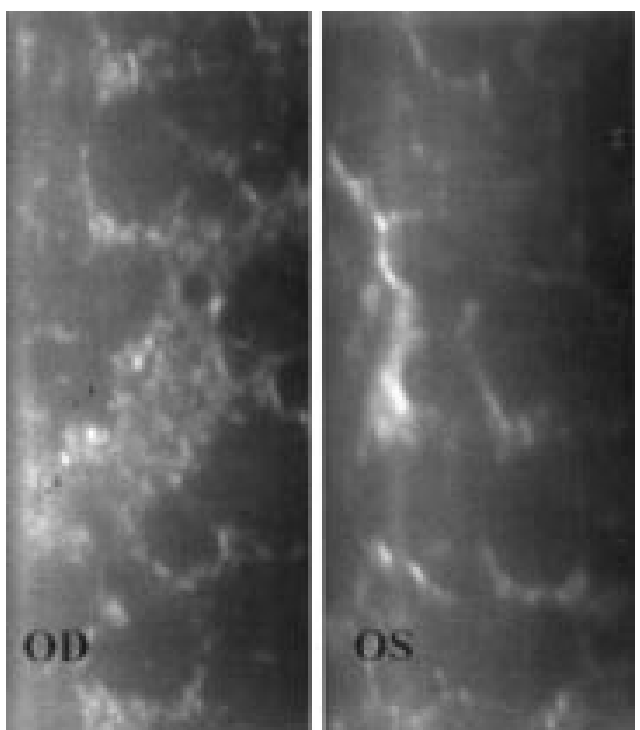

Figure 4 Patient 2. Specular microscopy in the right (OD) and left (OS) eyes in a case of bilateral corneal guttae and oedema (Fuchs'dystrophy).

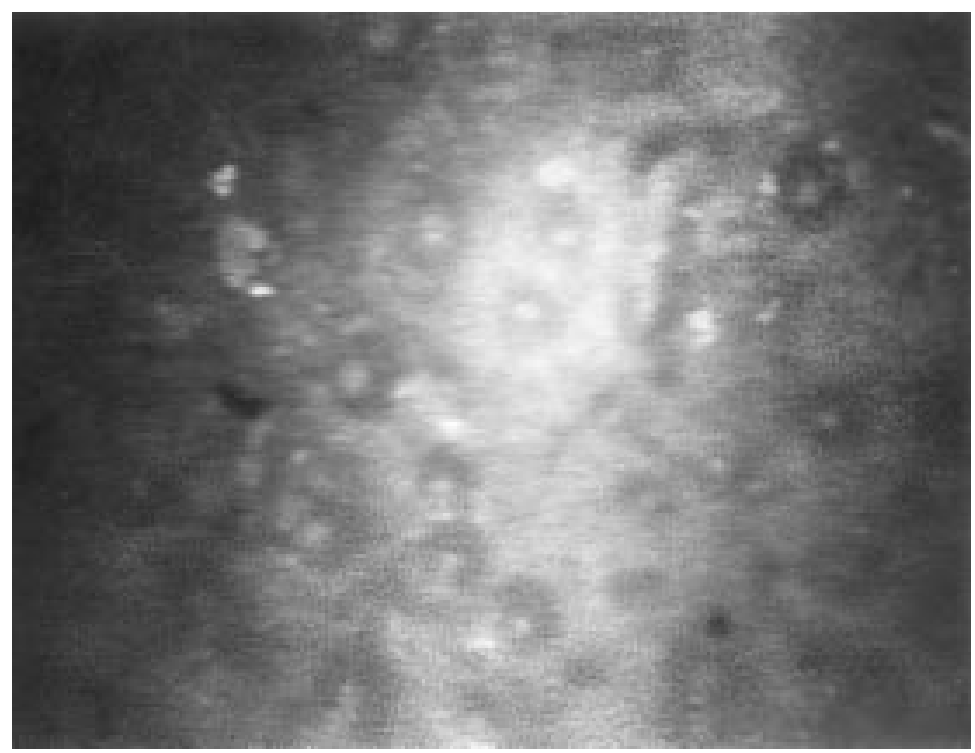

Figure 5 Patient 2. Confocal microscopy (magnification $\times 210$ ) of the right eye. Hyporeflective images with a central highlight were seen in the endothelium.

\section{Materials and methods}

Seven eyes of four consecutive patients with clinical signs of cornea guttata or Fuchs' dystrophy were prospectively studied. Specular microscopy using a non-contact microscope (Topcon SP 2000 P, Tokyo, Japan) was performed. Patients then underwent in vivo confocal microscopic examination of their eyes. We used a prototype white light tandem scanning confocal microscope (LSU Eye Center, New Orleans, LA, USA). The instrument utilised a $24 \times / 0.60$ contact objective and allowed optical sectioning of the cornea with a depth of field of $12 \mu \mathrm{m}$. The images were captured using a video camera (CCD 200 E, Videoscope International, Washington DC, USA) and stored on S-VHS video tapes. Confocal and specular microscopic findings were analysed and compared.

\section{Results}

CASE 1

A 41 year old black male patient with a history of bilateral cornea guttata was examined. Familial and general medical history was unremarkable. The patient had been treated with hypertonic saline drops in the right eye. Best corrected visual acuity was $20 / 30$ in the right eye, and 20/20 in the left eye. Slit lamp examination of the right eye disclosed advanced beaten metal appearance of the corneal endothelium. In the left eye the endothelium showed moderate beaten metal appearance. Intraocular pressure was $16 \mathrm{~mm} \mathrm{Hg}$ in the right eye and $18 \mathrm{~mm} \mathrm{Hg}$ in the left eye. The remainder of the ocular examination was normal. Corneal thickness was measured by ultrasonic pachymetry. The right cornea measured $594 \mu \mathrm{m}$ versus $553 \mu \mathrm{m}$ in the left eye.

Specular microscopic examination (Fig 1) of the right cornea demonstrated large numbers of dark bodies. Between the dark bodies, the endothelial cells appeared hyperreflective and their boundaries could not be identified. In the left eye the same alterations were demonstrated, however, to a much lesser extent. There were fewer dark bodies and the endothelial cells were well individualised. They presented polymegathism and pleomorphism.

In the left eye (Fig 2), confocal microscopy demonstrated endothelial cells with polymegathism, pleomorphism, and few hyporeflective round images containing occasionally a central highlight. The right eye (Fig 3) presented more significant alterations. At the level of the endothelium, hyporeflective round images were found in larger size and number. Between them, the endothelial cells appeared hyperreflective and could not be identified individually.

\section{CASE 2}

A 72 year old white woman with progressive decrease of vision in both eyes was referred for evaluation. She had a long term history of cornea guttata. However, corneal oedema was never documented. Besides a medically well controlled hypothyroidism, no other abnormalities were reported. She mentioned a sister diagnosed with Fuchs' endothelial dystrophy. 


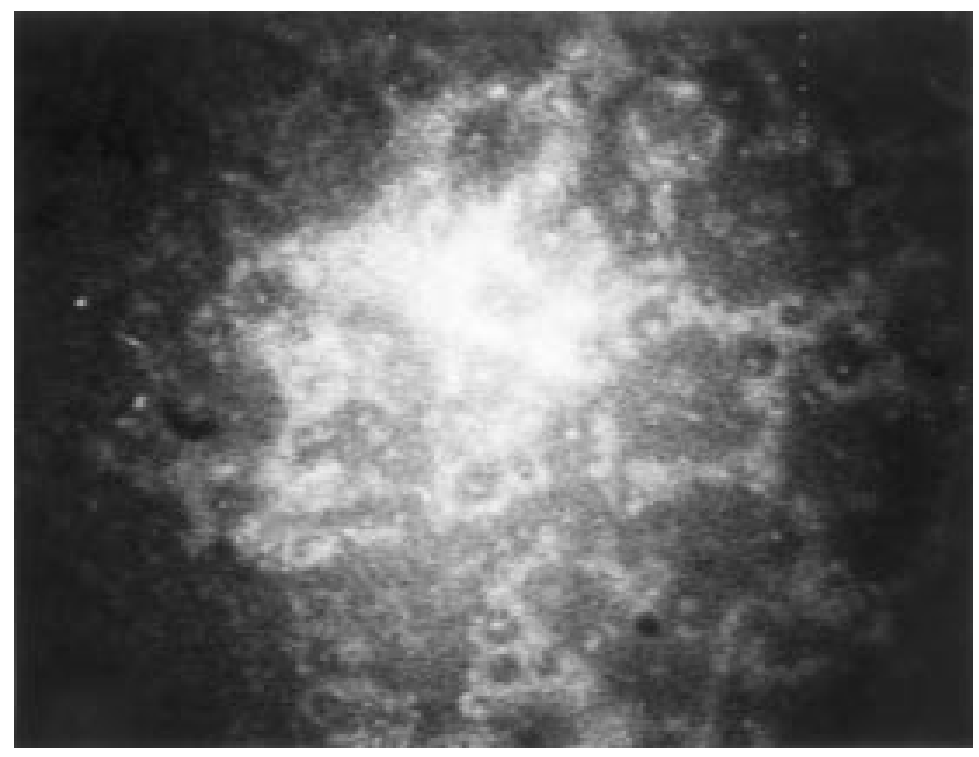

Figure 6 Patient 2. Confocal microscopy (magnification $\times 210$ ) of the left eye. Confluent hyporeflective images with a sporadic central highlight were demonstrated.

Best corrected vision was $20 / 60$ and $20 / 80$ in the right and left eye, respectively. In both eyes, corneal thickness appeared increased on slit lamp examination, and mild epithelial oedema was noted in the left eye. Both eyes presented significant beaten metal appearance at the level of the endothelium and moderate nuclear cataract. Intraocular pressure was $14 \mathrm{~mm} \mathrm{Hg}$ in both eyes. The remainder of the examinations was within normal ranges. Corneal thickness measured by ultrasonic pachymetry was 632 $\mu \mathrm{m}$ and $643 \mu \mathrm{m}$ in the right and left eye, respectively.

Specular microscopy demonstrated in both eyes large numbers of dark bodies in the corneal endothelium (Fig 4). In the right eye, endothelial cells with pleomorphism and polymegathism could be recognised. Otherwise and especially in the left eye, the endothelial cells appeared compressed between the dark bodies.

Confocal microscopy detected in both eyes the presence of multifocal hyporeflective areas surrounded by hyperreflective endothelial cells (Figs 5 and 6). The density of the hyporeflective images varied from one area to another in both eyes.

CASE 3

A 81 year old white female patient was evaluated for progressive decrease of vision in the right eye. Cornea guttata was documented in both eyes 5 years earlier, when the left eye underwent uncomplicated phacoemulsification and intraocular lens implantation. She was anticoagulated with warfarin because of past episodes of brain strokes. She denied topical medications. Best corrected vision was $20 / 70$ in the right eye and 20/40 in the left eye. Both eyes presented with beaten metal endothelial appearance. The right eye had a relatively advanced cataract and the left eye was pseudophakic. Intraocular pressure was $16 \mathrm{~mm} \mathrm{Hg}$ and $17 \mathrm{~mm} \mathrm{Hg}$ for the right and left eye, respectively. Both eyes had moderate nonexudative age related macular degeneration.

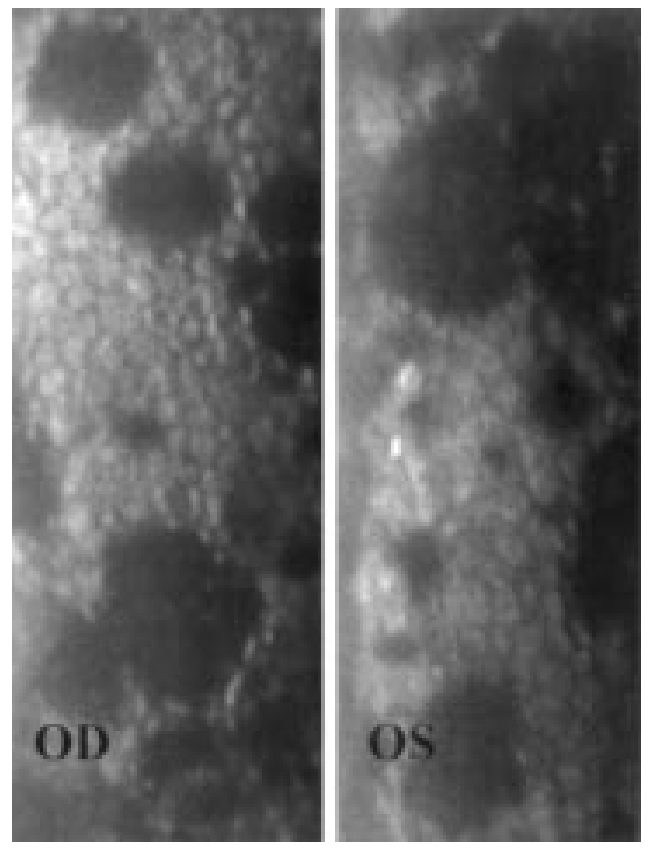

Figure 7 Patient 3. Specular microscopy in a case of bilateral cornea guttata. OD indicates right eye and $O S$ indicates left eye.

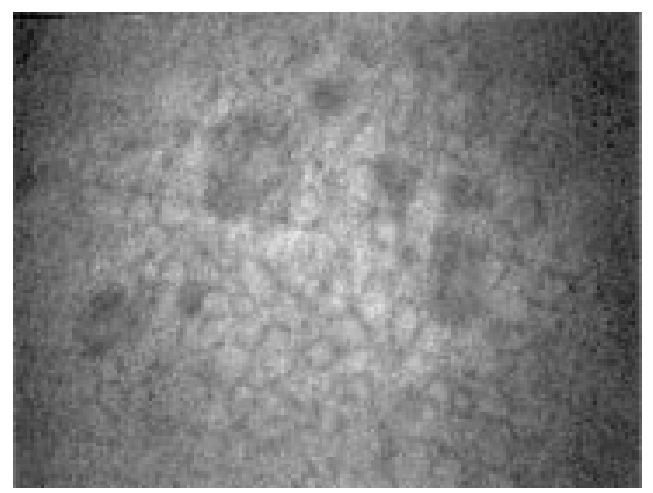

Figure 8 Patient 3. Confocal microscopy (magnification $\times 210$ ) of the right eye. Hyporeflective images were seen in the endothelium.

Corneal thickness measured $568 \mu \mathrm{m}$ in the right eye and $589 \mu \mathrm{m}$ in the left eye.

Specular microscopy of the endothelium demonstrated confluent dark bodies in both eyes (Fig 7). The endothelial cells presented polymegathism and pleomorphism.

Confocal microscopy revealed the presence of hyporeflective round images with an occasional central highlight among pleomorphic endothelial cells of varying size in both corneas (Figs 8 and 9). In each eye, areas with few hyporeflective images could be found, while other areas demonstrated numerous and confluent hyporeflective images.

CASE 4

A 74 year old white male patient was examined. His left eye underwent functional penetrating keratoplasty with cataract extraction and intraocular lens implantation 3 years earlier for Fuchs' endothelial dystrophy and senile cataract. Histological examination of the corneal button confirmed the diagnosis of Fuchs' endothelial dystrophy. He denied any topical treatments. Best corrected visual acuity 


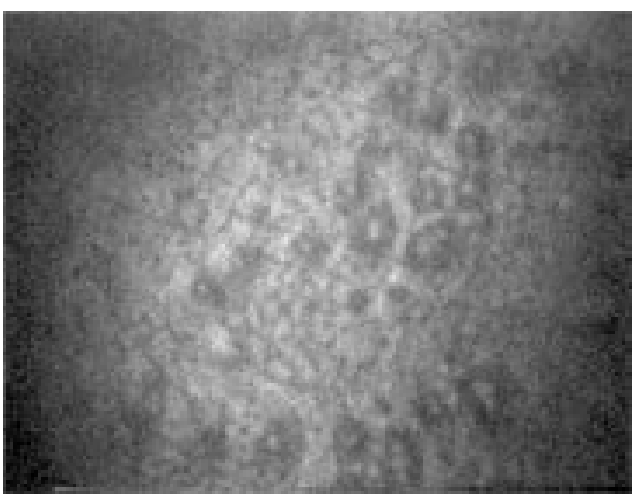

Figure 9 Patient 3. Confocal microscopy (magnification $\times 210$ ) of the left eye. Hyporeflective images with a central highlight were demonstrated in the endothelium.

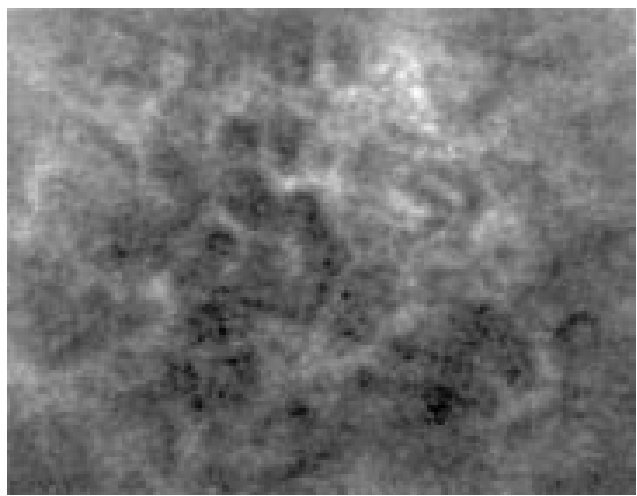

Figure 10 Patient 4. Confocal microscopy (magnification $\times 210)$ revealed the presence of confluent hyporeflective images in the endothelium. Hyperreflective endothelial cells were found between the lesions. The hyperreflective image on the upper part of the photograph is compatible with fibrous changes. Specular microscopy in this case of Fuchs' endothelial dystrophy was prevented by corneal oedema.

was $20 / 200$ and $20 / 40$ in the right and left eye, respectively. Slit lamp examination of the right eye disclosed significant beaten metal appearance of the endothelium, increased stromal thickness, and epithelial oedema. The right eye also had relatively advanced nuclear cataract. The left eye had a clear graft and was pseudophakic. Intraocular pressure was $11 \mathrm{~mm} \mathrm{Hg}$ in the right eye and $12 \mathrm{~mm} \mathrm{Hg}$ in the left eye. No other abnormalities were noted. Further examination was performed only in the right eye. Corneal thickness by ultrasonic pachymetry was $662 \mu \mathrm{m}$.

Non-contact specular microscopy was prevented by corneal oedema.

On the other hand, confocal microscopy easily revealed the presence of confluent hyporeflective images with hyperreflective endothelial cells between them (Fig 10). Hyperreflective images compatible with fibrous tissue were also seen.

\section{Discussion}

To the best of our knowledge, the confocal microscopic appearance of corneal guttae has not been reported so far. Recognition of corneal guttae is important, since they are the hallmark of cornea guttata and of Fuchs' dystrophy. Kaufman et al previously reported a case of Fuchs' endothelial dystrophy and documented the presence of larger and irregu- larly shaped endothelial cells on confocal microscopy. ${ }^{14}$ Presence of guttae was not demonstrated. Their findings were compatible with cell loss and may be associated with various disorders, and therefore, were not characteristic of Fuchs' dystrophy.

The dark round bodies with occasional central white reflex found on specular microscopy are typical of guttae. ${ }^{15} 16$ They were noticeably similar to the confocal microscopic findings. Therefore, by comparison with the specular microscopic findings, we suggest that the hyporeflective images with an occasional central highlight seen on confocal microscopy correlate with guttae. The similarity of the appearances of guttae on confocal and specular microscopy is not surprising, since both techniques detect reflected light from biological samples. At the level of the guttae, light is transmitted, whereas at the surrounding stroma endothelium interface it is reflected. Consequently, the lesions appear hyporeflective. At the apex of the guttae, reflection of light may result in a central highlight.

Guttae may be seen in various conditions. They include interstitial keratitis, ${ }^{17}$ corneal macular dystrophy, ${ }^{18}$ and posterior polymorphous dystrophy. ${ }^{19}$ It is unclear whether they would appear similar to our findings when examined with the confocal microscope. However, differential diagnosis is straightforward, since each of these disorders is associated with typical anterior segment changes. The same applies to pseudoguttae, which are guttae-like images caused by endothelial oedema that may appear in cases of corneal inflammation and disappear upon resolution of the underlying disease. ${ }^{20} 21$

The illustrations show that the specular image is clearer than the confocal image. This is probably due to the fact that the confocal images were frame grabbed. The image of the endothelial cells is clearer when viewed in real time. As illustrated in patient 4 and reported previously by Kaufman et al, confocal microscopy is not precluded by corneal oedema. ${ }^{14}$ This represents the main advantage of this technology over specular microscopy. Furthermore, since the severity of the endothelial alterations may be variable from one area to another within the same eye, we think that the confocal microscope, because of its ability to scan the entire cornea, allows for a better qualitative appreciation of the endothelium. It should be noted that contact specular microscopy, unlike the non-contact specular microscope used in this study, also has the ability to scan the entire cornea. Like specular microscopy, confocal microscopy does not enable prediction of the natural history of cornea guttata. It does not indicate whether eyes with corneal endothelial guttae are susceptible to develop corneal oedema. With both techniques endothelial polymorphism and pleomorphism could be detected. Specular microscopy remains, nevertheless, an invaluable diagnostic tool in evaluating the corneal endothelium. Compared with confocal microscopy, it is easier to use and does not require a learning curve. Furthermore, the quality of the images 
is rarely dependent on the patient's ability to remain still.

Cornea guttata and Fuchs' dystrophy are rarely a diagnostic dilemma, and most of the time are readily diagnosed simply by slit lamp examination. Based on our experience, we think that confirmation of the diagnosis is best accomplished by specular microscopy, because it is easier to use. However, confocal microscopy is a worthwhile alternative, especially in cases of corneal oedema.

The authors do not have any commercial or proprietary interes in the products or companies cited in this article. The authors do not have any financial interest or receive payment as a consultant, reviewer, or evaluator.

This study was supported in part by US Public Health Servce grants EY00346 (SCK), EY02580 (HEK), and EY02377 (HEK) from the National Eye Institute, National Institutes of Health, Bethesda, MD; Department of the Army, Cooperative Agreement DAMD17-93-V-3013. (This does not necessarily reflect the position or the policy of the government, and no official endorsement should be inferred) (RWB, HEK); an unrestricted departmental grant from Research to Prevent Blindness, Inc, New York City; Société Académique Vaudoise (AGYC), Switzerland; the Swiss National Research Foundation (AGYC), Switzerland; and Verrey Foundation (AGYC), Switzerland.

1 Waring GO 3rd, Rodrigues MM, Laibson PR. Corneal dystrophies. II. Endothelial dystrophies. Surv Ophthalmol 1978;23:147-68.

2 Krachmer JH, Purcell JJ Jr, Young CW, et al. A study of sixty-four families with corneal endothelial dystrophy. Arch Sixty-four families with corneal

3 Beuerman RW. Confocal microscopy: Into the clinic. Cornea 1995;14:1-2.

4 Cavanagh HD, Petroll WM, Alizadeh H, et al. Clinical and diagnostic use of in vivo confocal microscopy in patient with corneal disease. Ophthalmology 1993;100:1444-54.

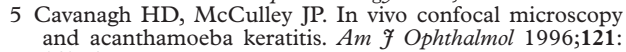
$207-8$
6 Chew SJ, Beuerman RW, Assouline M, et al. Early diagnosis of infectious keratitis with in vivo real time confocal microscopy. CLAO f 1992;18:197-201.

7 Chiou AGY, Cadez R, B $\div$ hnke M. Diagnosis of Dieffenbachia induced corneal injury by confocal microscopy. $\mathrm{Br} \mathcal{F}$ Ophthalmol 1997;81:168-9.

8 Florakis GJ, Moazami G, Schubert H, et al. Scanning slit confocal microscopy of fungal keratitis. Arch Ophthalmol 1997;115:1461-3.

9 Kaufman SC, Chew SJ, Capps SC, et al. Confocal microscopy of corneal penetration by tarantula hairs. Scanning 1994;16:312-5.

10 Kaufman SC, Beuerman RW, Goldberg D. A new form of primary, localized, corneal amyloidosis: A case report with confocal microscopy. Metab Pediatr Syst Ophthalmol 1995; 18:1-4.

11 Pfister DR, Cameron JD, Krachmer JH, et al. Confocal microscopy findings of acanthamoeba keratitis. Am f Ophthalmol 1996;121:119-28.

12 Shah GK, Pfister D, Probst LE, et al. Diagnosis of microsporidial keratitis by confocal microscopy and the chromatrope stain. Am $\mathcal{F}$ Ophthalmol 1996;121:89-91.

13 Sutphin JE, Kantor AL, Mathers WD, et al. Evaluation of infectious crystalline keratitis with confocal microscopy in a case series. Cornea 1997;16:21-6.

14 Kaufman SC, Beuerman RW, Kaufman HE. Diagnosis of advanced Fuchs' endothelial dystrophy with the confocal microscope. Am f Ophthalmol 1993;116:652-3.

15 Laing RA, Leibowitz HM, Oak SS, et al. Endothelial mosaic in Fuchs' dystrophy. A qualitative evaluation with the specular microscope. Arch Ophthalmol 1981;99:80-3.

16 Bigar F, Schimmelpfennig B, Hurzeler R. Cornea guttata in donor material. Arch Ophthalmol 1978;96:653-5.

17 Waring GO, Font RL, Rodrigues MM, et al. Alterations of Descemet's membrane in interstitial keratitis. $A m \quad \mathcal{F}$ Ophthalmol 1976;81:773-85.

18 Pouliquen Y, Dhermy P, Renard G, et al. Combined macular dystrophy and cornea guttata: An electron microscopic study. Albrecht Von Graefes Arch Klin Exp Ophthalmol 1980; 212:149-58.

19 Cibis GW, Krachmer JA, Phelps CD, et al. The clinical spectrum of posterior polymorphous dystrophy. Arch Ophthalmol 1977;95:1529-37.

20 Brooks AM, Grant GB, Gillies WE. The identification of corneal guttae. Cornea 1991;10:249-60.

21 Krachmer JH, Schnitzer JI, Fratkin J. Cornea pseudoguttata: a clinical and histopathologic description of endothelial cell edema. Arch Ophthalmol 1981;99:1377-81. 